\title{
Variations in the high-mobility group-A2 gene (HMGA2) are associated with idiopathic short stature
}

\author{
Ileana Fusco', Deepak Babu', Simona Mellone1, Nadia Barizzone', Flavia Prodam², Antonella Fanelli', Ranjit Muniswamy', \\ Antonella Petri², Simonetta Bellone², Gianni Bona ${ }^{2}$ and Mara Giordano'
}

BACKGROUND: Several association studies confirmed highmobility group-A2 gene (HMGA2) polymorphisms as the most relevant variants contributing to height variability. Animal models and deletions in humans suggest that alterations of HMGA2 might be relevant in causing short stature. Together, these observations led us to investigate the involvement of HMGA2 in idiopathic short stature (ISS) through an association study and a mutation screening.

METHODS: We conducted an association study (155 ISS patients and 318 normal stature controls) with three HMGA2 single-nucleotide polymorphisms (SNPs) (SNPs rs1042725, rs7968682, and rs7968902) using a TaqMan-based assay. The patients were then analyzed by direct sequencing and multiplex ligation-dependent probe amplification (MLPA) to detect point mutations and genomic micro-rearrangements.

RESULTS: Considering a recessive model, an OR value $>1$ was observed for genotypes rs7968682 TT (Odds ratio $(\mathrm{OR})=1.72$, confidence interval (Cl): 1.14-2.58) and rs1042725 TT (OR = 1.51, Cl: 1.00-2.28) in accordance to the effect exhibited by the single alleles in the general population. None of the patients carried possibly causative HMGA2 mutations.

CONCLUSION: Besides the already known role in determining variability in human height, HMGA2 polymorphisms also contribute to susceptibility to ISS. Moreover, we here report the first mutation screening performed in ISS concluding that HMGA2 has not a significant impact on the monogenic form of ISS.

$\mathrm{H}$ eight is a highly variable polygenic trait with an estimated $80-90 \%$ of the variation explained by a genetic component $(1,2)$. The variability has a normal distribution in the population with the extremes that go far beyond the normal variation, considering "normal" a height comprised between -2 and +2 SD. Short stature is the condition characterized by a height lower than 2 SD below the mean for a given age, sex, and population group and represents the most common reason for pediatric consultation. Idiopathic short stature (ISS) describes a heterogeneous group of phenotypes consisting of unidentified causes of short stature, presenting the following common criteria: normal size for gestational age at birth, normal body proportions, no evidence of pituitary hormone deficiency, no evidence of chronic organic disease, no psychiatric disease or severe emotional disturbance and normal food intake. According to this definition, it is estimated that approximately $80 \%$ of all children referred for short stature will be labeled as ISS at the end of the diagnostic work-up $(3,4)$.

Mutations and deletions in the short stature homeobox gene (SHOX) located within the pseudoautosomal region 1 (PAR1) and representing the best known genetic cause of ISS have been detected in the $7-15 \%$ of ISS patients $(5,6)$. However in only a minority of the ISS patients, a genetic defect has been identified.

A contribution to the understanding of the genetic etiology of short stature come from genome-wide association studies (GWAS). In the past years, several GWAS identified hundreds of common variants contributing to height determination in the general population. The most recent and comprehensive GWAS was reported by Visscher et al. (7) that analyzed the combined results of 79 GWAS using data from 253,288 individuals. They showed that less than 10,000 single-nucleotide polymorphisms (SNPs) can account for $36 \%$ of height and identified 697 SNPs clustered in 423 loci reaching a genome wide significance. Chan et al. (8) observed that the lower extreme of the phenotype distribution was shorter than what expected based on the polygenic model. Different models might explain this observation. For example, deprivation, malnutrition, comorbidity with other disorders or other environmental conditions might disturb the polygenic inheritance at this extreme of the curve. Moreover high penetrance rare variants might account for at least a portion of the extreme phenotypes whereas another part of them might be determined by a combination of quite common variants in susceptibility genes.

Loci that have been replicated across several GWAS in different populations likely represent the major genetic determinants influencing height. Common variants within and close the 3'UTR of the HMGA2 (NM_003483) have been repeatedly associated with childhood and adult height determination in

\footnotetext{
'Department of Health Sciences, Laboratory of Human Genetics, University of Eastern Piedmont, Novara and IRCAD (Interdisciplinary Research Center of Autoimmune Diseases), Novara, Italy; ${ }^{2}$ Department of Health Sciences, University of Eastern Piedmont, Paediatrics Unit, Novara, Italy. Correspondence: Mara Giordano (mara.giordano@med.uniupo.it)

Received 4 May 2015; accepted 5 August 2015; advance online publication 2 December 2015. doi:10.1038/pr.2015.225
} 
multiple populations from different geographical areas showing the strongest association $\left(<10^{-10}\right)$ in several studies (9-12). These polymorphisms might also play a role in the susceptibility to ISS in patients that do not carry high penetrance mutations.

A role of HMGA2 in ISS is strongly supported by the observation that rare microdeletions on $12 \mathrm{q} 14$ including HMGA2 in the critical region have been detected in patients with short stature as a common feature $(13,14)$. Interestingly, a submicroscopic intragenic deletion disrupting only HMGA2 has been identified in a boy with idiopathic proportionate short stature, without any other abnormalities (15). Moreover, lossof-function hmga2 mutations in mice resulted in the pygmy phenotype, characterized by extremely decreased fat levels and pre- and postnatal growth failure (16)..

The high-mobility group A (HMGA) family comprises several proteins: HMGAla, HMGA1b, HMGA1c produced by the alternative splicing of the HMGA1 gene, and HMGA2 encoded by the HMGA2 gene. HMGAs, expressed mainly during embryonic development, act exclusively as architectural proteins by binding the minor groove of AT-rich DNA sequences. They have no transcriptional activity per se, but they are able to alter the chromatin structure by interacting with the transcription machinery, and thus they can negatively or positively regulate the transcriptional activity of several genes (17).

In order to assess the role of HGMA2 in the determination of ISS, we compared the frequency of three HGMA2 SNPs in a group of patients and normal stature controls of Italian origin. We also performed a mutation analysis of the HMGA2 gene to identify rare variants with high effect that might be related to monogenic forms of short stature.

\section{RESULTS}

\section{Association Study}

The SNPs rs7968682, rs7968902, and rs1042725 were genotyped in the 155 ISS patients and 318 controls. These SNPs were selected as their contribution to height determination was replicated across different GWAS (9-12). Allele and genotype frequencies were consistent with those expected from Hardy-Weinberg equilibrium.

The frequency of the rs7968682T allele was significantly higher in ISS patients than in controls $(P=0.012$, OR: 1.44; Table 1, allelic model). A similar result was observed when adding sex as covariate to the logistic regression analysis $(P=0.017, \mathrm{OR}=1.41)$. Considering a recessive model, an OR value $>1$ was observed for genotypes rs7968682 TT $(\mathrm{OR}=1.72$, $P=0.00622$, Table 1$)$ and $\mathrm{rs} 1042725 \mathrm{TT}(\mathrm{OR}=1.51, P=0.037)$.
Stratifying the analysis for gender both rs7968682 and rs1042725 were significantly associated in the female group (rs7968682 TT: $\mathrm{OR}=2.01, P=0.0163, \mathrm{~T}: \mathrm{OR}=1.71, P=0.013$; rs1042725: TT OR $1.77 P=0.05 \mathrm{~T}: \mathrm{OR}=1.65 ; P=0.020$ ) whereas in the males only a nonsignificant trend was observed (rs7968682 TT: OR = $1.48 \mathrm{~T}: \mathrm{OR}=1.24 ; \mathrm{rs} 1042725$ TT OR 1.31 $T: \mathrm{OR}=1.0 .7$; all these values were not significant).

Due to linkage disequilibrium (D' ranging from 0.82 to 0.98 and $r^{2}$ from 0.57 to 0.78 ), the three SNPs generated only six different haplotypes (Table 2). Only two rare combinations, namely CGG and TTT, showed a different distribution in patients and controls. The common haplotype carrying the two associated variations (namely TGT) although present with higher frequency in patients, showed only a borderline statistical significant difference $(P=0.055)$ between patients and controls. Thus, none of the allele combinations allowed to better define an associated haplotype and the rs7968682T remains the most associated variant.

\section{Mutation Screening}

All the exons and the splicing junctions of HMGA2 were sequenced in the 155 short stature patients in order to find causative mutations. No mutation was identified. Already known polymorphisms, all located outside the coding regions, were detected with a frequency similar to that reported in public database (data not shown). In one patient, a synonymous heterozygous exon variation, namely Ser105Ser (exon 5, rs201860923) was identified, already reported in the Exome Variant Server, with a frequency of $0.07 \%$ (Broad Institute of MIT and Harvard, Cambridge, MA). In order to see whether it fell in unknown putative splicing regulatory elements, an in silico analysis was performed by using ESEfinder 3.0 (Cold Spring Harbor Laboratory, NY) and Spliceview (CNR-ITB Milan, Italy). This variation did not alter the score of putative exonic splicing enhancer sequences compared to the threshold value and did not create any novel splice site.

The presence of deletions and duplications of the single exons and the whole $H M G A 2$ gene region was investigated through multiplex ligation-dependent probe amplification. None of the patients carried copy number variations at this locus.

\section{DISCUSSION}

HMGA2 polymorphisms at the 3'UTR have been found to influence variation in human height. Although the SNPs were not always the same, the effect of the associated alleles was replicated in the same direction in different populations. In

Table 1: Association analysis on the three SNPs ( $n=155$ ISS, $318 \mathrm{HC}$ )

\begin{tabular}{|c|c|c|c|c|c|c|c|c|c|c|c|c|}
\hline \multirow[b]{2}{*}{ SNP } & \multirow[b]{2}{*}{ RA } & \multicolumn{2}{|c|}{ RAF } & \multicolumn{3}{|c|}{ Allelic model } & \multicolumn{3}{|c|}{ Dominant model } & \multicolumn{3}{|c|}{ Recessive model } \\
\hline & & ISS & $\mathrm{HC}$ & $P$ value & OR & $95 \% \mathrm{Cl}$ & $P$ value & OR & $95 \% \mathrm{Cl}$ & $P$ value & OR & $95 \% \mathrm{Cl}$ \\
\hline rs1042725 & $\mathrm{T}$ & 0.66 & 0.60 & 0.064 & 1.31 & $0.97-1.75$ & 0.48 & 1.21 & $0.68-2.19$ & 0.037 & 1.51 & $1.00-2.28$ \\
\hline rs7968682 & $\mathrm{T}$ & 0.69 & 0.61 & 0.012 & 1.44 & $1.07-1.95$ & 0.33 & 1.32 & $0.73-2.39$ & 0.0062 & 1.72 & $1.14-2.58$ \\
\hline
\end{tabular}

The significant values are shown in bold.

$\mathrm{Cl}$, confidence interval; $\mathrm{HC}$, healthy controls; ISS, idiopathic short stature; OR, Odds ratio; RA, risk allele; RAF, risk allele frequecy; SNP, single-nucleotide polymorphism. 
Table 2 Haplotype association

\begin{tabular}{lccccc}
\hline Haplotype & $\begin{array}{c}\text { ISS } \\
\text { frequency }\end{array}$ & $\begin{array}{c}\mathrm{HC} \\
\text { frequency }\end{array}$ & $\begin{array}{l}P \text { value } \\
\text { OR }\end{array}$ & $95 \% \mathrm{Cl}$ \\
\hline TGT & 0.641 & 0.576 & 0.0555 & 1.31 & $0.98-1.76$ \\
CTG & 0.270 & 0.306 & 0.2611 & 0.84 & $0.61-1.15$ \\
CGG & 0.029 & 0.065 & $\mathbf{0 . 0 2 2 3}$ & 0.43 & $0.19-0.94$ \\
CTT & 0.039 & 0.031 & 0.4841 & 1.31 & $0.59-2.87$ \\
TGG & 0.010 & 0.023 & 0.1825 & 0.43 & $0.1-1.62$ \\
TTT & 0.010 & 0.000 & $\mathbf{0 . 0 1 5 9}$ & - & - \\
\hline
\end{tabular}

Haplotypes are indicated with the single-nucleotide polymorphism alleles in this order: rs1042725, rs7968902, rs7968682.

The significant values are shown in bold.

$\mathrm{Cl}$, confidence interval; HC, healthy controls; ISS, idiopathic short stature; OR, Odds ratio.

Japanese, for example, Takeshita et al. (18) reported the association with rs7968902: homozygotes for the T-allele were on average shorter (about $6 \pm 1.2 \mathrm{~cm}$ ) than homozygotes for the G-allele. In Caucasians and in Chinese, the association with rs1042725 was confirmed (Yang et al. (19)) in the same direction with each copy of the rs1042725C allele estimated to be associated with an increase in height of $0.6 \mathrm{~cm}$. An association study conducted in tall individuals (20) showed that the rs1042725C allele was significantly associated with tall stature: individuals carrying this variant yield a significantly increased odd of being tall $(\mathrm{OR}=1.53,95 \% \mathrm{CI}$ : $1.02-2.28 ; P=0.03)$.

Altogether these studies identified rs1042725C, rs7968902T, and rs7968682 $G$ as "height increasing" alleles and rs1042725T, rs7968902G, and rs7968682T as "height decreasing" alleles in the general population. These findings encouraged us to assess whether these SNPs influenced the susceptibility to ISS, by comparing their frequency in short stature patients and controls. The increased OR of 1.72 and 1.51 observed for ISS subjects carrying the rs7968682TT and rs1042725TT genotypes, respectively, suggested a recessive effect of these polymorphisms (Table 1). Although the effect of these variants is stronger and significant in the females, there is no sufficient statistic power to assess that these SNPs confer a gender-specific risk).

It is difficult to assess which SNP is directly responsible for the association because of the strong linkage disequilibrium between the two SNPs $\left(0.57<r^{2}<0.79\right)$.

The 3 HMGA2 SNPs are located within and close to the HMGA2 3'-UTR that contains multiple binding sites for the tumor suppressor microRNA let-7 $(21,22)$. None of the SNP falls within a predicted miRNA binding site but it has been recently shown that the sequence context around the miRNA site is also important, as RNA secondary structure and RNA binding protein can affect miRNA function (23). The rs 1042725 (localized at chr12:66,358,347), located in the proximity (13 bp apart) of a let-7 putative binding site (predicted by Targetscan 6.2, Whitehead Institute for Biomedical Research, Cambridge, MA) might directly or indirectly influence the binding of a miRNA although this hypothesis can be demonstrated only through a functional test.

Deletions in disorders characterized by growth failure and evidence in mice (16) strongly suggested an involvement of HMGA2 high-penetrance mutations in ISS. In a previous study conducted on isolated growth hormone deficiency patients, only an intron variation in one patient out of 105 was identified (24). The present work is the first reported mutation screening performed on HMGA2 in ISS and contrarily to our expectation neither mutations nor copy number variation were identified thus suggesting that HMGA2 has not a significant impact on the monogenic form of short stature.

In conclusion, our findings strengthen the role of the HMGA2 polymorphisms in the determination of height and show that specific genotypes (rs7968682TT and/or rs1042725TT) contribute to the multifactorial forms of ISS.

\section{METHODS}

\section{ISS Subjects}

One hundred and fifty-five unrelated individuals (82 males and 73 females) with a median age at diagnosis of 10.9 y (5.1-17.8) were recruited by the Unit of Paediatrics of the Department of Health Sciences of Novara over a period of $6 \mathrm{y}$ and were included in the genetic study. The subjects were referred to the clinical center because they had a height $\leq-2$ SD score (SDS) or a height $\leq 1.8$ SDS in combination with a height velocity over $1 \mathrm{y}<-2$ SDS using the criteria of Tanner-Whitehouse (25). The mean height was $-2.43 \pm 0.51$ SDS ranging from -4.6 and -1.8 SDS. The growth velocity was $-1.4 \pm 2.1$ SDS.

Skeletal maturation was estimated as bone age (radius, ulna, and short bone) with the TW2 method by a pediatric endocrinologist (26). The mean delay in bone age relative to chronological age was $2.57 \pm 1.86 \mathrm{y}$.

They were all evaluated for GH serum levels after two provocative tests (with arginine or clonidine or insulin or glucagon or with GHRH+arginine). None of the patients included in this study showed $\mathrm{GH}$ deficiency. In all the subjects, deletions/duplications and point mutations in the SHOX gene were previously excluded as the cause of short stature during routine genetic testing.

Three hundred and eighteen normal stature healthy pediatric individuals (174 males and 144 females) matched for age and sex not tested for GH secretion levels were included in the association study as controls. A written informed consent was obtained from the patient's and control's parents, as they were all aged less than 18 . The study was approved by the Institutional Review Board (Review Board of "Azienda Ospedaliera Universitaria Maggiore della Carità").

\section{Genotyping}

Genomic DNA of short stature patients and controls was extracted from the blood samples by standard salting-out method. SNP genotyping was performed with Taqman technology and StepOnePlus Real-Time PCR System (Applied Biosystems, Foster City, CA) to study the three SNPs rs1042725, rs7968682, and rs7968902, in ISS patients and normal stature matched controls.

\section{Sequence Analysis}

The DNA was amplified by polymerase chain reaction (PCR) using primers designed to specifically amplify the coding region and the intron/exon boundaries of the gene (Supplementary Table S1 online), using the software Primer3 Input (Version 0.4.0, Whitehead Institute for Biomedical Research). The primer pair specificity was controlled with the Basic local Alignment Search Tool (National Centre for Biotechnology Information, Bethesda, MD). The PCR reaction was carried out with the GoTaq Flexi DNA polymerase (Promega Corporation Fitchburg, Wisconsin) in a $15-\mu$ l reaction volume, at an annealing temperature of $58^{\circ} \mathrm{C}$ for exons 2 and 4 and $62^{\circ} \mathrm{C}$ for exons 1,3 , and 5 .

The PCR products were visualized on a $2 \%$ agarose gel and purified using ExoSAP-IT (Affymetrix, Santa Clara, CA) enzymatic PCR clean up system. The purified products were then sequenced with the Big Dye Terminator kit (Applied Biosystems) and the automatic sequencer ABI PRISM 3100 Genetic Analyzer (Applied Biosystems). 


\section{Multiplex Ligation-Dependent Probe Amplification}

Multiplex ligation-dependent probe amplification for all exons of $H M G A 2$ gene was performed by means of a commercial kit according to the manufacturer's protocol (SALSA MLPA P323 CDK4-HMGA2MDM2, MRC Holland, Amsterdam, The Netherlands).

\section{Statistical Analysis}

The analysis set consisted of the mentioned 155 patients and 318 healthy controls all fully genotyped for the three SNPs and successfully sequenced for the 5 exons of the gene. Basic association analysis with no covariates added was performed for allelic, dominant, and recessive models with PLINK 1.9 software (Center for Human Genetic Research, Massachusetts General Hospital, and the Broad Institute of Harvard \& MIT). Dominant and recessive models were calculated on the basis of genotypic counts: homozygous for the risk allele vs. the other two classes pooled for the recessive model, homozygous for the risk allele plus heterozygous vs. homozygous for the protective allele for the dominant model.

Linkage disequilibrium between the three SNPs was calculated on the whole Italian sample set (both patients and controls). Linkage disequilibrium calculation and haplotype frequencies determination was performed with the Haploview software (Center for Human Genetic Research, Massachusetts General Hospital, and the Broad Institute of Harvard \& MIT). Basic association analysis at the haplotype level was performed with Haploview.

\section{SUPPLEMENTARY MATERIAL}

Supplementary material is linked to the online version of the paper at http:// www.nature.com/pr

\section{ACKNOWLEDGMENTS}

We are grateful to all the patients and their families. A special thanks to Patricia Momigliano-Richiardi for helpful comments and critical reading of the manuscript.

\section{STATEMENT OF FINANCIAL SUPPORT}

This work was supported by Cariplo Foundation, Milano Italy. I.F, D.B., and R.M. were PhD fellow of Dottorato di Ricerca in Medicina Molecolare supported by University of Eastern Piedmont, Novara, Italy

Disclosure: None of the authors declare any conflict of interest.

\section{REFERENCES}

1. Silventoinen K, Sammalisto S, Perola M, et al. Heritability of adult body height: a comparative study of twin cohorts in eight countries. Twin Res 2003;6:399-408.

2. Visscher PM, Macgregor S, Benyamin B, et al. Genome partitioning of genetic variation for height from 11,214 sibling pairs. Am J Hum Genet 2007;81:1104-10

3. Cohen P, Rogol AD, Deal CL, et al.; 2007 ISS Consensus Workshop participants. Consensus statement on the diagnosis and treatment of children with idiopathic short stature: a summary of the Growth Hormone Research Society, the Lawson Wilkins Pediatric Endocrine Society, and the European Society for Paediatric Endocrinology Workshop. J Clin Endocrinol Metab 2008;93:4210-7.

4. Pedicelli S, Peschiaroli E, Violi E, Cianfarani S. Controversies in the definition and treatment of idiopathic short stature (ISS). J Clin Res Pediatr Endocrinol 2009;1:105-15.

5. Benito-Sanz S, Barroso E, Heine-Suñer D, et al. Clinical and molecular evaluation of SHOX/PAR1 duplications in Leri-Weill dyschondrosteosis (LWD) and idiopathic short stature (ISS). J Clin Endocrinol Metab 2011;96:E404-12.

6. Chen J, Wildhardt G, Zhong Z, et al. Enhancer deletions of the SHOX gene as a frequent cause of short stature: the essential role of a $250 \mathrm{~kb}$ downstream regulatory domain. J Med Genet 2009;46:834-9.

7. Wood AR, Esko T, Yang J, et al.; Electronic Medical Records and Genomics (eMEMERGEGE) Consortium; MIGen Consortium; PAGEGE Consor- tium; LifeLines Cohort Study. Defining the role of common variation in the genomic and biological architecture of adult human height. Nat Genet 2014;46:1173-86.

8. Chan Y, Holmen OL, Dauber A, et al. Common variants show predicted polygenic effects on height in the tails of the distribution, except in extremely short individuals. PLoS Genet 2011;7:e1002439.

9. Weedon MN, Lettre G, Freathy RM, et al.; Diabetes Genetics Initiative; Wellcome Trust Case Control Consortium. A common variant of HMGA2 is associated with adult and childhood height in the general population. Nat Genet 2007;39:1245-50.

10. Weedon MN, Lango H, Lindgren CM, et al.; Diabetes Genetics Initiative; Wellcome Trust Case Control Consortium; Cambridge GEM Consortium. Genome-wide association analysis identifies 20 loci that influence adult height. Nat Genet 2008;40:575-83.

11. Lango Allen H, Estrada K, Lettre G, et al. Hundreds of variants clustered in genomic loci and biological pathways affect human height. Nature 2010;467:832-8.

12. Lettre G, Jackson AU, Gieger C, et al.; Diabetes Genetics Initiative; FUSION; KORA; Prostate, Lung Colorectal and Ovarian Cancer Screening Trial; Nurses' Health Study; SardiNIA. Identification of ten loci associated with height highlights new biological pathways in human growth. Nat Genet 2008;40:584-91

13. Mari F, Hermanns P, Giovannucci-Uzielli ML, et al. Refinement of the $12 \mathrm{q} 14$ microdeletion syndrome: primordial dwarfism and developmental delay with or without osteopoikilosis. Eur J Hum Genet 2009;17:1141-7.

14. Lynch SA, Foulds N, Thuresson AC, et al. The 12q14 microdeletion syndrome: six new cases confirming the role of HMGA2 in growth. Eur J Hum Genet 2011;19:534-9.

15. Buysse K, Reardon W, Mehta L, et al. The 12q14 microdeletion syndrome: additional patients and further evidence that HMGA2 is an important genetic determinant for human height. Eur J Med Genet 2009;52:101-7.

16. Zhou X, Benson KF, Ashar HR, Chada K. Mutation responsible for the mouse pygmy phenotype in the developmentally regulated factor HMGIC. Nature 1995;376:771-4

17. Cleynen I, Van de Ven WJ. The HMGA proteins: a myriad of functions (Review). Int J Oncol 2008:32:289-305.

18. Takeshita H, Fujihara J, Soejima M, et al. Confirmation that SNPs in the high mobility group-A2 gene (HMGA2) are associated with adult height in the Japanese population; wide-ranging population survey of height-related SNPs in HMGA2. Electrophoresis 2011;32:1844-51.

19. Yang TL, Guo Y, Zhang LS, et al. HMGA2 is confirmed to be associated with human adult height. Ann Hum Genet 2010;74:11-6.

20. Hendriks AE, Brown MR, Boot AM, Oostra BA, Drop SL, Parks JS. Genetic variation in candidate genes like the HMGA2 gene in the extremely tall. Horm Res Paediatr 2011;76:307-13.

21. Lee YS, Dutta A. The tumor suppressor microRNA let-7 represses the HMGA2 oncogene. Genes Dev 2007;21:1025-30.

22. Papaioannou G, Inloes JB, Nakamura Y, Paltrinieri E, Kobayashi T. let-7 and miR-140 microRNAs coordinately regulate skeletal development. Proc Natl Acad Sci USA 2013;110:E3291-300.

23. Haas U, Sczakiel G, Laufer SD. MicroRNA-mediated regulation of gene expression is affected by disease-associated SNPs within the 3'-UTR via altered RNA structure. RNA Biol 2012;9:924-37.

24. Gorbenko del Blanco D, de Graaff LC, Posthouwer D, Visser TJ, Hokken-Koelega AC. Isolated GH deficiency: mutation screening and copy number analysis of HMGA2 and CDK6 genes. Eur J Endocrinol 2011;165:537-44.

25. Tanner JM, Whitehouse RH. Clinical longitudinal standards for height, weight, height velocity, weight velocity, and stages of puberty. Arch Dis Child 1976;51:170-9.

26. Tanner JM, Whitehouse RH, Cameron N, Marshall WA, Healy WA, Goldstein H. Assessment of Skeletal Maturity and Prediction of Adult Height (TW2 Method). San Diego, CA: Academic Press, 1988. 